\title{
Enhancement and improvement of the mechanical properties of aluminum extruded products by mathematical analysis and simulation process
}

\begin{abstract}
This paper investigates a technique for solving the problems of the aluminum extrusion process, and improving the mechanical properties of the products produced by this method through a smart design, simulation and Mathematical Analysis by using F.E.M. For this purpose, the general F.E.A. Software ABAQUS was used to set up the finite element model of the warm aluminum extrusion in two dimensions (3D). Also, an iterative procedure was carried out using MATLAB at each iteration. The model was formulated as a nonlinear model. The inputs to this model were: the product geometry and its materials specifications such as density, rigidity, elasticity, thermal conductivity, and the required analytical steps. An axisymmetrical (3D) geometric model of the tooling and billet was constructed for the analysis. Data obtained from the F.E model included die-work piece contact pressure, effective stress and strain and material deformation velocity. The correlation between the calculated and collected data from (FEA) was established in this research. Then the billet and die stresses, temperature and the ram speed that closely matched with the strain rates for the desired quality were obtained.
\end{abstract}

Keyword: Aluminum extrusion; Analysis; FEM simulation 